\title{
Benchmarking en responsabilidad social corporativa. El caso de las empresas gallegas ${ }^{1}$
}

\section{Corporate social responsibility benchmarking. The case of galician firms}

\author{
ENCARNACión GonZÁlez VÁZqueZ² \\ $M^{\text {a }}$ Elisa Alén González ${ }^{3}$ \\ LORENZO RODRÍGUEZ COMESAÑA ${ }^{3}$
}

Universidad de Vigo (España)

Recibido el 15 de abril de 2010 y aceptado el 29 de junio de 2010

$\mathrm{N}^{\circ}$ de clasificación JEL: M14

DOI: $10.5295 /$ cdg. 100226 eg

\section{Resumen:}

En el presente trabajo se realiza una revisión del concepto de responsabilidad social. Posteriormente se analizan las posibilidades y problemas de la utilización del benchmarking en RSE haciendo un análisis de las últimas investigaciones que desarrollaron algún método de benchmarking. A partir de ese análisis se propone un indicador homogéneo que evalúa 68 aspectos relacionados con los diferentes stakeholders implicados en RSE. También se recoge información sobre la importancia otorgada por las empresas encuestadas a estos aspectos. Los resultados obtenidos para cada uno de los 5 sectores considerados muestran las áreas en las que el trabajo realizado en RSE es mayor y aquellas en las que es necesario mejorar.

Palabras clave:

benchmarking, responsabilidad social corporativa, stakeholders

\section{Abstract:}

In this paper we review the concept of corporate social responsibility. Subsequently we analyze the possibilities and problems of the use of benchmarking in CSR by analyzing the latest research that had developed a method of benchmarking. From this analysis we propose a homogeneous indicator that assesses 68 aspects related to the various stakeholders involved in CSR. It also provides information on the importance attached by respondents to these aspects. The results for each of the 5 sectors considered show the areas in which the work in CSR is greatest and others where improvement is needed.

\section{Keywords:}

benchmarking, corporate social responsibility, stakeholder perspective

\footnotetext{
${ }^{1}$ Esta investigación ha sido posible gracias a la financiación de la Xunta de Galicia al proyecto PGIDIT05CSO30001PR.

${ }^{2}$ Universidad de Vigo, Facultad de CCEE, Lagoas Marcosende, s/n 36200. Vigo. Email:egzlez@uvigo.es

${ }^{3}$ Universidad de Vigo, Facultad de CCEE y Turismo, Campus Universitario, s/n 32004 Ourense. Email: alen@ uvigo.es loroco@uvigo.es
} 


\section{INTRODUCCIÓN}

El rol de la empresa en la sociedad ha sido un problema objeto de discusión desde la mitad del siglo pasado. Debido a este creciente interés social en el comportamiento responsable de las empresas, surge el concepto de responsabilidad social corporativa (en adelante RSC). En este contexto, la RSC formaliza de alguna manera la idea de que el conjunto de responsabilidades que las empresas están dispuestas a aceptar excede sus obligaciones puramente legales y económicas (Igalens y Gond, 2005). Esto es debido al hecho de que el entorno en el cual las empresas llevan a cabo sus actividades es también social, político y ecológico en naturaleza. Por ello, la RSC puede proporcionar un marco general para estructurar el uso responsable del poder corporativo y el entorno social.

A pesar de la importancia creciente de la RSC, no existe consenso cuando se trata de definirla. En este sentido, se pueden encontrar numerosas definiciones de RSC tanto en artículos académicos, como en informes de empresas y en documentos de organismos públicos, pero no hay y probablemente no haya una definición única y precisa, porque su contenido y aplicación puede variar de un país a otro, puede cambiar a lo largo del tiempo y de las empresas. Esto no sólo es confuso, sino también es un indicador de la inherente debilidad del concepto (Argandoña y Von Weltzien, 2009). La dimensión ética está presente en la mayoría de las definiciones de RSC, aunque frecuentemente de manera implícita, con expresiones como "compromiso de las empresas en la gestión de su papel en la sociedad" (International Chamber of Commerce, 2002), "contribución a una sociedad mejor y a un medioambiente más limpio" (European Commission, 2001). Por otra parte, las definiciones al menos implican (si no lo establecen explícitamente) que las organizaciones son relacionales y de este modo están conectadas (intencionalmente o no) con sus stakeholders mucho más allá de sus accionistas y empleados. En este sentido, la RSC es el resultado implícito o explicito de una reflexión sobre la naturaleza de la firma, su rol en la sociedad y sus relaciones con sus stakeholders internos y externos. Es decir, la empresa en sus actuaciones debe tener en cuenta tanto a los agentes que influyen como a los que son influidos por ella, a la sociedad en general: local, nacional, universal y futura (de la Cuesta y Valor, 2003).

En opinión de Kolodinsky et al. (2010), la RSC es el deber ético de una organización por encima de sus obligaciones legales y fiduciarias con sus accionistas, la cual considera sensiblemente y gestiona de manera efectiva sus impactos en las relaciones y en el entorno tanto a nivel interno, como externo. Ese deber o responsabilidades son retrospectivas (relativas a acciones previas u omisiones) y prospectivas (relacionadas con comportamientos futuros), e incluyen la disposición a actuar de determinada manera (responsabilidad) y a dar cuentas de esas acciones precisamente a la sociedad o la comunidad en la cual actúan. De acuerdo con este punto de vista, la primera razón para que una empresa sea responsable debe ser su obligación ética, por encima de otro argumento o ventaja, ya sea esta material o reputacional.

En este contexto, la participación de una empresa en RSC puede ser explicada utilizando varios argumentos motivacionales. Estas motivaciones pueden ser clasificadas en estratégicas y altruistas (Campbell et al., 1999; Lantos, 2001) posicionando de este modo los motivos económicos para la participación en RSE (por ejemplo, Donaldson y Preston, 1995; Hillman y Keim, 2001), junto a los morales (por ejemplo, Joyner y Payne, 2002). En términos prácticos, tanto las evidencias científicas (Margolis y Walsh, 2003; Orlitzky et 
al., 2003; Waddock y Graves, 1997), como la reacción de los consumidores (McWilliams y Siegel, 2001), han señalado a las empresas que su participación en la RSC es probable que sea recompensada, resultando en un mejor rendimiento. La participación en RSC puede mejorar las relaciones con los distintos stakeholders (McWilliams y Siegel, 2001), con lo que se reduce el riesgo empresarial (Boutin-Dufresne y Savaria, 2004). Por estas y otras razones, el valor estratégico de la RSC está siendo cada vez más reconocido (Porter y Kramer, 2002).

La importancia estratégica alcanzada por la RSC ha generado una expansión de las expectativas destinadas a asegurar un seguimiento de su desarrollo. Esta inquietud se vincula con la necesidad de establecer indicadores que permitan identificar esfuerzos de medición, evaluación, mejoras y comunicación de sus resultados e impactos mejorando la transparencia. Pero esta medición entraña grandes dificultades, siendo un problema investigado tanto por académicos, como por profesionales (Panayiotou et al., 2009). Así, una manera adecuada de mejorar la transparencia de los esfuerzos en RSC que hacen las empresas es realizando acciones de benchmarking por entes independientes. La construcción de un índice que dé idea de la contribución de las empresas a través de un número, puede clarificar la posición de empresas individuales y mejorar la comparabilidad de los esfuerzos en RSC. La publicación de este índice podría de manera potencial impulsar el mecanismo de la reputación y proporcionar una ventaja competitiva a las empresas que profundicen activamente en valores sociales y ecológicos. Esto podría proveer a otras empresas de un fuerte incentivo para integrar la RSC en la estrategia de la empresa (Graafland, 2002).

El benchmarking crea un entorno competitivo inter-empresas que actúa como un incentivo para mejorar la RSC. El incrementar los niveles de transparencia a través del benchmarking es una herramienta poderosa que muestra los fallos de la empresa al público, afecta a las decisiones de inversores socialmente responsables y también es un riesgo potencial para su soporte financiero (Lee y Kohler, 2010).

Por eso en el presente trabajo, se establece como objetivo principal la elaboración de un índice (benchmark) que permita evaluar el grado de desarrollo de la RSC en las empresas gallegas, de modo que permita comparaciones entre empresas y también entre distintos sectores. Para ello se comienza haciendo una revisión de la literatura específica sobre benchmarking en RSC.

\section{BENCHMARKING EN RSC}

El benchmarking ha sido una herramienta de los negocios en su lucha para conseguir un conjunto de elevados estándares de desempeño en cualquier área de la empresa. Si el benchmarking puede ser utilizado de manera efectiva en áreas de responsabilidad pública, no hace falta imaginar que es de gran utilidad en las áreas de responsabilidad social y comportamiento ético. Por tanto, las empresas deben llevar a cabo benchmarking, tanto competitivo como funcional, obteniendo información de los competidores y empresas fuera de la industria que están intentando desarrollar informes exhaustivos y comprensivos sobre responsabilidad pública (Joyner y Raiborn, 2005).

En este sentido, las principales ventajas que se derivan de la aplicación del benchmarking en RSC son (Graafland et al, 2004: 138-140): (1) Fomenta la transparencia. A través 
del benchmarking las empresas tienen un índice de sus acciones y logros, que permite a los stakeholders juzgar cómo de responsable es una compañía específica; (2) Mejora la medición. Cuando la puntuación está disponible es más fácil para los stakeholders confrontar la empresa con sus acciones, es decir, posibilita el juicio sobre si la empresa mejoró su responsabilidad respecto al año anterior. A la compañía le permite identificar sus debilidades; (3) Posibilita la comparación entre empresas. En el sentido de que una empresa con una elevada puntuación es más responsable que otra con una puntuación más baja; (4) La simplicidad del procedimiento. Todo el mundo puede juzgar el desempeño en RSC de una empresa analizando una o más puntuaciones; (5) Enfoque sistemático para juzgar la contribución de la empresa y no depende de incidentes. Un benchmarking sistemático de todos los aspectos relevantes del comportamiento de una empresa puede proporcionar una visión mucho mas equilibrada de la calidad de su política de RSC; (6) Realizado por entidades externas independientes garantiza una visión más objetiva que la presentación de los resultados de RSC por la propia empresa; y (7) Requiere que la empresa recopile la información necesaria para la construcción del índice.

Sin perjuicio de las ventajas del benchmarking, el método ha sido criticado por expertos en ética, así como por algunos directivos. Se han puesto de manifiesto algunos problemas que afectan sobre todo a la parte cuantitativa del método, pero la dificultad de medida no debe ser usada como una excusa para no llevar a cabo la evaluación. Los principales problemas de benchmarking identificados en RSC son (Graafland et al, 2004: 140-142): (1) Monismo y medición. Para sumar varios valores se debe asumir una hipótesis de monismo y de cuantificabilidad de dichos valores. Esas asunciones son sin embargo, cuestionables; (2) Intenciones. La práctica del benchmark no tiene en cuenta las intenciones, lo que es importante para la evaluación de una acción moral; (3) Subjetividad de la evaluación. Las acciones morales deberían poder ser valoradas, lo que en la mayoría de las ocasiones sólo puede ser realizado por el propio investigador; (4) Punto de vista de la preocupación ética. En la práctica no se considera que algunos stakeholders deberían cuidarse más que otros; (5) Contexto de la acción moral. En la práctica no se considera que una empresa no tiene el control total de algunas acciones y por tanto, no tiene que ser totalmente responsable de esas acciones; (6) Daños comunicativos. Se necesita la transparencia de la empresa. La compañía, sin embargo, puede no estar interesada en dar información sobre sus acciones morares debido a los posibles daños que esto pueda suponer en el estatus moral de la acción.

En la tabla 1 se recoge la síntesis de algunos estudios de benchmarking en RSC. En ella se puede observar como Krut y Munis (1998) elaboraron un índice cualitativo para medir la RSC, pero solamente utilizaron la dimensión medioambiental, focalizando su estudio en las empresas de dos sectores. Por su parte, Graves y sus colegas elaboraron siete medidas, una para cada stakeholder considerado (todos los stakeholders tienen el mismo peso). La información se recoge de varias fuentes y debido a que se encontraba en distintas unidades de medida los autores proceden a su estandarización. Dentro de cada dimensión la puntuación positiva y negativa en ítems distintos se compensan. Posteriormente, calculan una media no ponderada de las dimensiones para llegar a determinar el valor/índice de cada empresa. 
Tabla 1

Algunos métodos recientes de benchmarking

\begin{tabular}{|c|c|c|c|c|}
\hline & Centrado en & Cuantitativo / cualitativo & $\begin{array}{l}\text { Fuentes de } \\
\text { información }\end{array}$ & $\begin{array}{l}\text { Método de } \\
\text { ponderación }\end{array}$ \\
\hline $\begin{array}{l}\text { Krut y } \\
\text { Munis } \\
(1998)\end{array}$ & $\begin{array}{l}\text { Dimensión medio- } \\
\text { ambiental, } 19 \\
\text { categorías }\end{array}$ & $\begin{array}{l}\text { Cualitativo (símbolos } \\
\text { tipográficos) }\end{array}$ & $\begin{array}{l}\text { Afirmaciones } \\
\text { en información } \\
\text { pública }\end{array}$ & No ponderado \\
\hline $\begin{array}{l}\text { Graves et } \\
\text { al (2002) }\end{array}$ & $\begin{array}{l}\text { Desempeño econó- } \\
\text { mico, social y ecoló- } \\
\text { gico, } 7 \text { categorías }\end{array}$ & $\begin{array}{l}\text { Cuantitativo (medidos } \\
\text { en una escala entre }-2 \\
y+2 \text { ) }\end{array}$ & $\begin{array}{l}\text { Fuentes } \\
\text { corporativas de } \\
\text { información, } \\
\text { cuestionarios, } \\
\text { periódicos, estudios } \\
\text { externos }\end{array}$ & $\begin{array}{l}\text { Ponderaciones } \\
\text { iguales }\end{array}$ \\
\hline $\begin{array}{l}\text { GRI } \\
(2002)\end{array}$ & $\begin{array}{l}\text { Desempeño econó- } \\
\text { mico, social y ecoló- } \\
\text { gico, } 5 \text { categorías }\end{array}$ & Cuantitativo & $\begin{array}{l}\text { Fuentes } \\
\text { corporativas de } \\
\text { información }\end{array}$ & $\begin{array}{l}\text { Ponderaciones } \\
\text { iguales }\end{array}$ \\
\hline $\begin{array}{l}\text { Vlek et al } \\
(2002)\end{array}$ & $\begin{array}{l}\text { Desempeño eco- } \\
\text { nómico, social y } \\
\text { ecológico }\end{array}$ & $\begin{array}{l}\text { Mezcla de un méto- } \\
\text { do de agregación de } \\
\text { valores y de juicios } \\
\text { cualitativos }\end{array}$ & $\begin{array}{l}\text { ¿Entrevistas o } \\
\text { cuestionarios? }\end{array}$ & $\begin{array}{l}\text { Diferentes } \\
\text { ponderaciones }\end{array}$ \\
\hline $\begin{array}{l}\text { Graafland } \\
\text { et al } \\
(2004)\end{array}$ & $\begin{array}{l}\text { Desempeño econó- } \\
\text { mico, social y ecoló- } \\
\text { gico, } 70 \text { categorías }\end{array}$ & $\begin{array}{l}\text { Cuantitativo (medido } \\
\text { en una escala con } \\
0,1 / 2,1)\end{array}$ & $\begin{array}{l}\text { Cuestionarios } \\
\text { contrastados } \\
\text { utilizando } \\
\text { información } \\
\text { pública }\end{array}$ & $\begin{array}{l}\text { Ponderaciones } \\
\text { basadas en las } \\
\text { respuestas de } \\
\text { las empresas y } \\
\text { las ONGs }\end{array}$ \\
\hline
\end{tabular}

Fuente: Adaptado de Graafland et al. (2004, p. 143)

El Global Reporting Initiative (GRI) ha proporcionado un modelo sistematizado de información sobre RSC estructurado según la triple dimensión del concepto de sostenibilidad. Se considera que los indicadores deben ser en la medida de lo posible: compatibles con criterios financieros, cuantificables, significativos para todas las empresas y sus stakeholders, y suficientemente precisos para permitir la evaluación del desempeño de la empresa. El GRI distingue los indicadores centrales que representan la expectativa mínima de un informe, lo que se aplica a todos los sectores y es del interés de la mayoría de los stakeholders; de los indicadores opcionales que son mas experimentales en naturaleza e interesantes para un stakeholder en particular (GRI, 2002). Los indicadores centrales deben ser agregados en cinco subtotales o menos.

El modelo abierto de Rusconi (2002) propone una estructura concéntrica que comprende cuatro niveles diferentes de valores sociales, cada uno parcialmente integrado en otro. El primer nivel calcula el valor directo añadido de la empresa y es obtenido de los estados financieros. El segundo nivel muestra los efectos positivos y negativos de la empresa, incluyendo los efectos externos como la polución por la que la empresa no paga o el desperdicio de know-how profesional. Estos efectos son calculados usando análisis coste-beneficio. El impacto económico directamente obtenido de los estados financieros 
de la empresa puede ser incluido como una subcategoría de valor añadido en el segundo nivel. El tercer nivel incluye aquellos efectos para los cuales el análisis coste-beneficio es teóricamente posible pero muy inapropiado o muy caro. El cuarto nivel incluye todos los aspectos que no pueden ser incluidos en los tres niveles anteriores.

Tencati y otros (2004) después de hacer una revisión de la literatura más relevante sobre RSC, tanto a nivel conceptual como a través del análisis de las propuestas hechas para su medición/evaluación, realizan una encuesta que les permite identificar los principales indicadores para cada stakeholder en el caso de empresas italianas. En base a esta investigación, proponen un conjunto de indicadores válidos para todo tipo de empresas (empresas públicas, grandes empresas y pequeñas y medianas empresas). Establecen tres niveles, de menor a mayor concreción: (1) Categorías, grupos de stakeholders que están específicamente afectados por grupos de indicadores; (2) Aspectos, áreas temáticas monitorizadas por grupos de indicadores de desempeño relacionados con una categoría concreta de stakeholder; y (3) Indicadores, medidas que pueden ofrecer información relacionada con un aspecto específico. Pueden ser usados para revisar o demostrar el desempeño organizacional. La información puede ser cualitativa, cuantitativa o económico-monetaria.

En la mayoría de los estudios revisados, la información sobre responsabilidad social proporciona indicadores de actuación respecto a los distintos grupos de interés, pudiendo ser ordenados según las tres dimensiones de la sostenibilidad, de forma independiente o interrelacionados. Así mismo, se pueden establecer dos niveles de indicadores, aquellos comunes presentes en prácticamente todas las empresas y los que sólo afectan a algunas de ellas. A otro nivel, los índices elaborados con estos indicadores varían en el grado en el cual no incluyen, o sólo incluyen un pequeño porcentaje de las empresas de un sector.

Teniendo en cuenta la revisión de la literatura realizada, se propone elaborar un índice que permita evaluar el grado de desarrollo de la RSC en las empresas gallegas. Para la elaboración de dicho índice se va a utilizar el enfoque stakeholder entendiendo que en el marco de la RSC la legitimidad para poder operar de una empresa ha de venir concedida por todos aquellos agentes o grupos de interés con los que se relaciona la organización (stakeholders). Bajo este paradigma se alcanza una optimización del valor que conduce a una dirección estratégica de la empresa con dimensiones que trascienden los resultados económicos e incluyen los sociales y medioambientales (Rodríguez Fernández, 2003).

\section{METODOLOGÍA}

La RSC puede ser investigada a través de varios métodos. Uno de ellos consiste en utilizar estudios de casos y análisis cualitativo como es el caso de la investigación llevada a cabo por Graafland (2002). La ventaja de estos métodos es que se obtiene mucha información sobre el entorno, las intenciones y los problemas de una empresa concreta. La principal desventaja es que no permite la comparación entre distintas empresas o que no se pueden generalizar conclusiones.

Otra posibilidad consiste en analizar información pública. Este método fue aplicado por Krut y Munis (1998). El principal inconveniente de este enfoque es que se asume que la transparencia de las empresas es suficientemente alta para asegurar que éstas no exageran su información pública. También es necesario tener en cuenta que la información pública de las empre- 
sas sobre RSC es relativamente escasa. Por eso en esta investigación el método de recogida de información utilizado ha sido el cuestionario. Por supuesto que también las empresas pueden no reflejar en las respuestas que dan al mismo su situación actual pero para intentar minimizar ese riesgo se ha invertido bastante tiempo en chequear las respuestas dadas al cuestionario utilizando información de los periódicos, informes anuales y otras fuentes públicas.

El cuestionario elaborado incluye la valoración de 68 aspectos de RSC así como la medida de la importancia que esos aspectos tienen para la empresa. Se han clasificado en función de los stakeholders con los que están relacionados, a saber: empleados, proveedores, clientes, sociedad, accionistas y competencia. Para cuantificar los resultados la mayoría de los aspectos ofrecían tres valoraciones posibles $(0,0.5$ y 1$)$. Este sistema es similar al método aplicado por Waddock y Graves (1997) y Graves et al. (2002). Algunos aspectos se evaluaron a través de preguntas abiertas para intentar aumentar la objetividad, posteriormente se recodificaron las respuestas a tres puntos. La medida de la importancia también se hizo a través de una escala de tres puntos (poco o nada importante - 0 , algo importante -0.5 , muy importante 1) y se agrupaba en conceptos en el caso de varias preguntas relacionadas siguiendo a Graafland et al. (2004).

Para determinar la población objeto de estudio se cogieron los 5 grandes sectores SIC por facturación en Galicia. Se excluyeron de la selección inicial las instituciones públicas y las entidades sin ánimo de lucro, ya que la naturaleza y los intereses de sus grupos de interés difieren significativamente de aquellos de las empresas con ánimo de lucro. Este modo de proceder permite la comparación entre diferentes empresas de un mismo sector, y además facilita la identificación de características o comportamientos específicos de un sector. Dentro de cada uno de esos grupos se seleccionaron las empresas más grandes en facturación hasta lograr una población de 300 empresas. Se eliminaron aquellas que se encontraban duplicadas, es decir, la misma empresa con varios códigos SIC. Al final se recogieron 109 encuestas válidas que conforman la muestra objeto de estudio.

Tabla 2

Descripción de la muestra

\begin{tabular}{lccc}
\hline \multicolumn{1}{c}{ Sector } & Sector SIC & $\mathrm{N}^{\circ}$ de empresas & Facturación 2006 \\
\hline Construcciones y contratas & 15,16 & 21 & 5.338 .793 .337 \\
Fabricantes & $20,21,22,23,24,27,28$, & 44 & 9.543 .119 .817 \\
Transporte & $30,32,33,34,35,37,39$ & & 52.200 .000 \\
Mayoristas & 44,47 & 2 & $9.710 .165 .816^{\prime} 33$ \\
Detallistas & 50,51 & 32 & 1.334 .786 .389 \\
\hline Total & $53,55,59$ & 10 & $25.979 .065 .319^{\prime} 33$ \\
\hline
\end{tabular}

Mayoritariamente la recogida de la información fue a través de entrevista personal, aunque algunos cuestionarios por imposibilidad de contactar una cita con algún responsable en la empresa, se recibieron a través del correo electrónico. 


\section{RESULTADOS}

Los resultados del estudio se presentan por stakeholder. Además de diferentes indicadores que evalúan de 0 a 1 el desempeño en distintos aspectos para cada grupo de interés relacionado con RSC, se analiza la importancia que las empresas encuestadas dan a estos aspectos.

\subsection{Clientes}

En la tabla 3 se recogen los valores alcanzados por cada indicador para la media del sector en la relación con sus clientes. Puesto que puede existir dispersión entre las empresas, se incluye también el valor de la mediana para poder tener una medida más exacta del desempeño de las empresas. Los datos reflejan que los aspectos en los que se ha trabajado más son en la calidad del producto y en los procedimientos para recoger quejas y sugerencias. Este patrón es válido para los distintos sectores analizados, si bien en el caso de los mayoristas se ha trabajado más en quejas que en calidad de producto. Estos resultados son coherentes si pensamos que estos son aspectos que tradicionalmente se recogen en cualquier norma de certificación de calidad, no así los elementos en los que el desempeño es menor como en desarrollo de productos innovadores. En este caso, el comportamiento es heterogéneo ya que en algunos sectores como el de fabricación ostenta un elevado valor, mientras que el desempeño es mínimo en construcciones y contratas o en el sector mayorista.

Si analizamos los datos de la tabla 4 podemos ver que para aquellos elementos en los que el desempeño era mayor la importancia también es alta, mientras que aquellos otros que hemos denominado 'alternativas sostenibles' han sido considerados por los diferentes sectores como menos relevantes. No se han detectado comportamientos diferentes en función del sector.

Tabla 3

Resultados de las relaciones con clientes (media y entre paréntesis mediana)

\begin{tabular}{|l|l|c|c|c|c|c|}
\hline $\begin{array}{c}\mathrm{N}^{\circ} \\
\text { Preg. }\end{array}$ & \multicolumn{1}{|c|}{ Aspecto } & $\begin{array}{c}\text { Constr. y } \\
\text { contratas }\end{array}$ & Fabric. & Transp.* & Mayor. & Minor. \\
\hline 3 & $\begin{array}{l}\text { Controlamos la calidad del } \\
\text { producto }\end{array}$ & $0.9737(1)$ & $1(1)$ & 1 & $0.875(1)$ & $0.95(1)$ \\
\hline 4 & $\begin{array}{l}\text { Comprobamos el impacto } \\
\text { medioambiental }\end{array}$ & $0.8571(1)$ & $0.8837(1)$ & 0.5 & $0.5323(0.5)$ & $0.75(1)$ \\
\hline 8 & $\begin{array}{l}\text { Alternativas sostenibles en el } \\
\text { surtido de productos }\end{array}$ & $\begin{array}{c}0.4048 \\
(0.5)\end{array}$ & $\begin{array}{c}0.5789 \\
(0.5)\end{array}$ & 0.75 & $0.5156(0.5)$ & 0.75 \\
\hline 9 & $\begin{array}{l}\text { Desarrolla productos } \\
\text { innovadores }\end{array}$ & $0.3095(0)$ & $0.8452(1)$ & 0.75 & $0.4531(0.5)$ & $0.5(0.5)$ \\
\hline 5 & $\begin{array}{l}\text { Existen procedimientos para } \\
\text { recoger quejas de clientes }\end{array}$ & $0.9524(1)$ & $0.9884(1)$ & 1 & $0.9531(1)$ & $0.95(1)$ \\
\hline 6 & No se usa publicidad engañosa & $0.5(0.5)$ & $0.7083(1)$ & 0.75 & $0.6452(1)$ & $0.95(1)$ \\
\hline 7 & $\begin{array}{l}\text { Se investiga sistemáticamente } \\
\text { la satisfacción del consumidor }\end{array}$ & $0.7381(1)$ & $0.8864(1)$ & 1 & $0.6875(1)$ & $0.9(1)$ \\
\hline
\end{tabular}

* La media y la mediana coinciden 
Tabla 4

Pesos por aspecto de las relaciones con clientes

\begin{tabular}{|l|l|c|c|c|c|c|}
\hline $\begin{array}{c}\text { Rel. } \\
\text { Preg. }\end{array}$ & \multicolumn{1}{|c|}{ Aspecto } & $\begin{array}{c}\text { Constr. y } \\
\text { contratas }\end{array}$ & Fabric. & Transp*. & Mayor. & Minor. \\
\hline 3 & $\begin{array}{l}\text { Calidad y seguridad de } \\
\text { producto }\end{array}$ & 1 & $1(1)$ & 1 & $\begin{array}{c}0.9531 \\
(1)\end{array}$ & $1(1)$ \\
\hline $4,8,9$ & $\begin{array}{l}\text { Ofrecemos alternativas } \\
\text { sostenibles }\end{array}$ & 0.7619 & $\begin{array}{c}0.7907 \\
(1)\end{array}$ & 0.5 & $\begin{array}{c}0.6406 \\
(0.5)\end{array}$ & $0.85(1)$ \\
\hline $5,6,7$ & Respeto por el cliente & 1 & $\begin{array}{c}0.9767 \\
(1)\end{array}$ & 1 & $1(1)$ & $1(1)$ \\
\hline
\end{tabular}

* La media y la mediana coinciden

\subsection{Empleados}

Para evaluar las distintas acciones con relación a los empleados se utilizaron en el cuestionario algunas preguntas de respuesta abierta que fue necesario recodificar $(26,27,28)$, para lo que se utilizó información secundaria y los datos de los cuartiles para cada sector.

En la tabla 5 se recogen los valores alcanzados por los distintos indicadores relacionados con los empleados. Se puede observar como entre los aspectos en los que el nivel de desempeño es mayor se encuentran el cumplimiento del horario laboral, con la excepción del sector de construcciones y contratas, la existencia de programas de formación, de programas sobre seguridad y salud laboral, o los esfuerzos llevados a cabo para prevenir accidentes. Es necesario considerar que algunos de estos aspectos son de obligado cumplimiento por las empresas por lo que es normal que el nivel que alcanza el indicador sea elevado. En el otro extremo se encuentran los aspectos menos regulados y que se entroncan más con el espíritu de RSC como es el caso del trato preferente a minorías extranjeras, a discapacitados o a mujeres, o la existencia de un dialogo activo con ONG's. Para la mayoría de las empresas y como tónica general de casi todos los sectores analizados las empresas no realizan este tipo de acciones, poniendo de manifiesto que la RSC todavía está en una fase muy iniciática.

El análisis de la importancia dada a los distintos aspectos y que se recoge en la tabla 6, ofrece sin embargo, un panorama alentador desde el momento en que toma valores bastante altos para la mayoría de ellos. Esto implicaría la existencia de un ambiente propicio a la implementación de estas políticas. Aún en este caso, se puede ver como los aspectos que tradicionalmente han sido objeto de regulación como la salud y seguridad laboral de los empleados son los más valorados, mientras que llama la atención que uno de los menos valorados sea la conciliación de la vida familiar y profesional, sobre todo en el caso del sector de fabricación. 
Tabla 5

Resultados de las relaciones con empleados (media y entre paréntesis mediana)

\begin{tabular}{|c|c|c|c|c|c|c|}
\hline $\begin{array}{c}\mathrm{N}^{\circ} \\
\text { Preg. }\end{array}$ & Aspecto & $\begin{array}{l}\text { Constr. y } \\
\text { contratas }\end{array}$ & Fabric. & Transp.* & Mayor. & Minor. \\
\hline 11 & Horario de trabajo & $0.5(0.5)$ & 1 & 1 & $1(1)$ & $1(1)$ \\
\hline 13 & $\begin{array}{l}\text { Trato preferente a minorías } \\
\text { extranjeras }\end{array}$ & $\begin{array}{l}0.1905 \\
(0)\end{array}$ & $\begin{array}{l}0.0732 \\
(0)\end{array}$ & 0.5 & $\begin{array}{c}0.2419 \\
(0)\end{array}$ & $0.25(0)$ \\
\hline 14 & $\begin{array}{l}\text { Trato preferente a } \\
\text { discapacitados }\end{array}$ & $\begin{array}{c}0.4048 \\
(0.5)\end{array}$ & $\begin{array}{l}0.3452 \\
(0)\end{array}$ & 0.25 & $\begin{array}{l}0.2742 \\
(0)\end{array}$ & $0.3(0)$ \\
\hline 25 & $\begin{array}{l}\text { Programas bienestar } \\
\text { trabajadores }\end{array}$ & $\begin{array}{l}0.3333 \\
(0.5)\end{array}$ & $\begin{array}{c}0.625 \\
(0.5)\end{array}$ & 0.25 & $\begin{array}{c}0.4516 \\
(0.5)\end{array}$ & $0.25(0)$ \\
\hline 21 & Facilita asistencia a formación & $0.881(1)$ & $0.907(1)$ & 0.75 & $\begin{array}{l}0.7969 \\
(1)\end{array}$ & $0.9(1)$ \\
\hline 29 & $\begin{array}{l}\text { Flexibilidad horaria y } \\
\text { permisos }\end{array}$ & $0.7(0.5)$ & $\begin{array}{c}0.7195 \\
(0.5)\end{array}$ & 0.5 & $\begin{array}{l}0.7656 \\
(1)\end{array}$ & $0.8(1)$ \\
\hline 22 & Diálogo activo con ONG's & $\begin{array}{l}0.0952 \\
(0) \\
\end{array}$ & $\begin{array}{c}0.0732 \\
(0) \\
\end{array}$ & 0.5 & $0.05(0)$ & $0.1(0)$ \\
\hline 15 & $\begin{array}{l}\text { Programas de ayuda a la } \\
\text { infancia }\end{array}$ & $0.119(0)$ & $0.5(0.5)$ & 0 & $\begin{array}{c}0.2344 \\
(0)\end{array}$ & $0.25(0)$ \\
\hline 12 & Trato preferente a mujeres & $\begin{array}{l}0.2381 \\
(0)\end{array}$ & $\begin{array}{c}0.2619 \\
(0) \\
\end{array}$ & 0.5 & $\begin{array}{c}0.3065 \\
(0)\end{array}$ & $0.25(0)$ \\
\hline 26 & $\begin{array}{l}\% \text { de mujeres en puestos } \\
\text { directivos }\end{array}$ & $0.5(0.5)$ & $\begin{array}{c}0.5769 \\
(0.5)\end{array}$ & 0.75 & $\begin{array}{c}0.5484 \\
(0.5)\end{array}$ & $\begin{array}{c}0.25 \\
(0.25)\end{array}$ \\
\hline 16 & Programas de formación & $\begin{array}{l}0.8571 \\
(1)\end{array}$ & $\begin{array}{l}0.9545 \\
(1)\end{array}$ & 0.75 & $0.875(1)$ & $1(1)$ \\
\hline 27 & $\begin{array}{l}\% \text { del salario gastado en } \\
\text { formación }\end{array}$ & $\begin{array}{l}0.6154 \\
(1)\end{array}$ & $\begin{array}{l}0.7885 \\
(1)\end{array}$ & 1 & $\begin{array}{l}0.5588 \\
(1)\end{array}$ & $0.5(0.5)$ \\
\hline 17 & $\begin{array}{l}\text { Programas sobre seguridad, } \\
\text { salud }\end{array}$ & $\begin{array}{c}0.9048 \\
(1)\end{array}$ & $\begin{array}{c}0.9091 \\
(1) \\
\end{array}$ & 1 & $\begin{array}{c}0.8125 \\
(1)\end{array}$ & $0.7(1)$ \\
\hline 28 & Ratio de bajas por enfermedad & $\begin{array}{c}0.8333 \\
(1) \\
\end{array}$ & $\begin{array}{c}0.8636 \\
(1) \\
\end{array}$ & 1 & $\begin{array}{l}0.7812 \\
(1) \\
\end{array}$ & $1(1)$ \\
\hline 18 & $\begin{array}{l}\text { Esfuerzo en prevenir } \\
\text { accidentes }\end{array}$ & $\begin{array}{l}0.9762 \\
(1)\end{array}$ & $\begin{array}{l}0.9773 \\
(1)\end{array}$ & 1 & $\begin{array}{l}0.9219 \\
(1)\end{array}$ & $0.95(1)$ \\
\hline 19 & $\begin{array}{l}\text { Rol del representante de los } \\
\text { trabajadores }\end{array}$ & $\begin{array}{c}0.7143 \\
(0.5)\end{array}$ & $0.75(1)$ & 0.75 & $\begin{array}{c}0.6250 \\
(0.75) \\
\end{array}$ & 0.85 (1) \\
\hline 23 & $\begin{array}{l}\text { Esfuerzo para prevenir abusos } \\
\text { en el trabajo }\end{array}$ & $\begin{array}{l}0.8056 \\
(1) \\
\end{array}$ & $\begin{array}{c}0.8295 \\
(1) \\
\end{array}$ & 1 & $\begin{array}{c}0.6607 \\
(0.5) \\
\end{array}$ & $\begin{array}{c}0.7222 \\
(1) \\
\end{array}$ \\
\hline 24 & $\begin{array}{l}\text { Esfuerzos para estimular el } \\
\text { compañerismo }\end{array}$ & $\begin{array}{l}0.8095 \\
(1)\end{array}$ & $\begin{array}{l}0.8068 \\
(1)\end{array}$ & 0.75 & $\begin{array}{l}0.7097 \\
(1)\end{array}$ & $0.75(1)$ \\
\hline 20 & $\begin{array}{l}\text { Investigaciones sistemáticas } \\
\text { en satisfacción laboral }\end{array}$ & $\begin{array}{c}0.4286 \\
(0.5)\end{array}$ & $\begin{array}{c}0.6163 \\
(0.5)\end{array}$ & 0.75 & $\begin{array}{c}0.3594 \\
(0.5)\end{array}$ & $\begin{array}{c}0.65 \\
(0.75)\end{array}$ \\
\hline
\end{tabular}

* La media y la mediana coinciden 
Tabla 6

Pesos por aspecto de las relaciones con empleados

\begin{tabular}{|l|l|c|c|c|c|c|}
\hline \multicolumn{1}{|c|}{$\begin{array}{c}\text { Rel. } \\
\text { Preg. }\end{array}$} & \multicolumn{1}{|c|}{ Aspecto } & $\begin{array}{c}\text { Constr. y } \\
\text { contratas }\end{array}$ & Fabric. & Transp* & Mayor. & Minor. \\
\hline $\begin{array}{l}11,14, \\
13,22\end{array}$ & $\begin{array}{l}\text { Defensa de los derechos } \\
\text { humanos }\end{array}$ & $\begin{array}{c}0.7619 \\
(1)\end{array}$ & $\begin{array}{c}0.7558 \\
(1)\end{array}$ & 1 & $\begin{array}{c}0.7188 \\
(1)\end{array}$ & $0.7(1)$ \\
\hline $\begin{array}{l}15,12, \\
26\end{array}$ & $\begin{array}{l}\text { Igualdad de oportunidades } \\
\text { para las mujeres }\end{array}$ & $\begin{array}{c}0.825(1) \\
21,25,\end{array}$ & $\begin{array}{c}0.7674 \\
(1)\end{array}$ & 1 & $\begin{array}{c}0.7812 \\
(1)\end{array}$ & $0.95(1)$ \\
\hline 29 & Ponciliación vida familiar y & $\begin{array}{c}0.6905 \\
(1)\end{array}$ & $\begin{array}{c}0.6744 \\
(0.5)\end{array}$ & 0.75 & $0.75(1)$ & $0.8(1)$ \\
\hline 16,27 & Formación de empleados & $\begin{array}{c}0.9524 \\
(1)\end{array}$ & $\begin{array}{c}0.8605 \\
(1)\end{array}$ & 0.75 & $\begin{array}{c}0.7969 \\
(1)\end{array}$ & $0.95(1)$ \\
\hline $\begin{array}{l}17,28, \\
18\end{array}$ & $\begin{array}{l}\text { Salud y seguridad laboral de } \\
\text { empleados }\end{array}$ & $\begin{array}{c}1(1) \\
19\end{array}$ & $\begin{array}{c}0.7674 \\
(1)\end{array}$ & 1 & $\begin{array}{c}0.6875 \\
(0.5)\end{array}$ & $1(1)$ \\
\hline $\begin{array}{l}\text { Participación de empleados } \\
23,24,\end{array}$ & Clima de trabajo & $\begin{array}{c}0.7143 \\
(1)\end{array}$ & $\begin{array}{c}0.9535 \\
(1)\end{array}$ & 0.75 & $\begin{array}{c}0.9375 \\
(1)\end{array}$ & $0.75(1)$ \\
\hline 20
\end{tabular}

* La media y la mediana coinciden

\subsection{Proveedores}

Las relaciones con proveedores son muy importantes en el contexto competitivo actual. Esto queda patente también en los datos recogidos en la tabla 7, puesto que todos los indicadores relacionados con este stakeholder alcanzan valores elevados. Los aspectos en los que las empresas han alcanzado un mayor nivel de desarrollo son en la calidad del producto y en la observancia de las condiciones laborales de los proveedores, aunque cada vez más se tiende a establecer estrategias de cooperación con ellos. La única excepción a esta última afirmación está en el sector minorista ya que este es el que menos está cooperando por el momento. Parece que las empresas en la actualidad no exigen que los proveedores estén certificados con ISO 9001, sobre todo para el sector de transportes.

Las valoraciones otorgadas a la importancia de los distintos aspectos de la relación con los proveedores reflejan un comportamiento simétrico al nivel de desempeño, ya que se considera que lo más importante es la calidad y seguridad del producto y el respeto al proveedor. Cabe resaltar que el sector que más bajas puntuaciones ha otorgado a la importancia de los distintos aspectos es el de transportes. Por otra parte, las empresas encuestadas consideraron poco importante tener información sobre la transparencia financiera del proveedor. 
Tabla 7

Resultados de las relaciones con proveedores (media y entre paréntesis mediana)

\begin{tabular}{|l|l|c|c|c|c|c|}
\hline $\begin{array}{c}\mathrm{N}^{\circ} \\
\text { Preg. }\end{array}$ & \multicolumn{1}{|c|}{ Aspecto } & $\begin{array}{c}\text { Constr. y } \\
\text { contratas }\end{array}$ & Fabric. & Transp.* & Mayor. & Minor. \\
\hline 38 & Requerimos certificación ISO & $\begin{array}{c}0.5238 \\
(0.5)\end{array}$ & $\begin{array}{c}0.6364 \\
(0.5)\end{array}$ & 0.25 & $\begin{array}{c}0.4355 \\
(0.5)\end{array}$ & $\begin{array}{c}0.4 \\
(0.25)\end{array}$ \\
\hline 31 & $\begin{array}{l}\text { Controlamos la calidad del } \\
\text { producto }\end{array}$ & $\begin{array}{c}0.9286 \\
(1)\end{array}$ & $\begin{array}{c}0.9773 \\
(1)\end{array}$ & 1 & $\begin{array}{c}0.7812 \\
(1)\end{array}$ & $0.9(1)$ \\
\hline 32 & $\begin{array}{l}\text { Controlamos la seguridad } \\
\text { medioambiental }\end{array}$ & $\begin{array}{c}0.8571 \\
(1)\end{array}$ & $\begin{array}{c}0.8295 \\
(1)\end{array}$ & 0.5 & $\begin{array}{c}0.5968 \\
(1)\end{array}$ & $0.7(1)$ \\
\hline 36 & $\begin{array}{l}\text { Sancionamos si las condiciones } \\
\text { medioambientales son malas o se } \\
\text { incumplen }\end{array}$ & $\begin{array}{c}0.8095 \\
(1)\end{array}$ & $\begin{array}{c}0.6829 \\
(1)\end{array}$ & 1 & $\begin{array}{c}0.7333 \\
(1)\end{array}$ & $0.7(1)$ \\
\hline 33 & $\begin{array}{l}\text { Controlamos las condiciones } \\
\text { laborales }\end{array}$ & $\begin{array}{c}0.8811 \\
(1)\end{array}$ & $\begin{array}{c}0.6744 \\
(1)\end{array}$ & 1 & $\begin{array}{c}0.5484 \\
(0.5)\end{array}$ & $\begin{array}{c}0.55 \\
(0.75)\end{array}$ \\
\hline 37 & $\begin{array}{l}\text { Existen sanciones en caso de } \\
\text { malas condiciones laborales }\end{array}$ & $\begin{array}{c}0.9(1) \\
0.775 \\
(1)\end{array}$ & 1 & $\begin{array}{c}0.7742 \\
(1)\end{array}$ & $0.8(1)$ \\
\hline 39 & $\begin{array}{l}\text { Controlamos transparencia } \\
\text { financiera proveedor }\end{array}$ & $\begin{array}{c}0.6905 \\
(0.5)\end{array}$ & $\begin{array}{c}0.7209 \\
(1)\end{array}$ & 0.5 & $\begin{array}{c}0.6452 \\
(0.5)\end{array}$ & $\begin{array}{c}0.7 \\
(0.75)\end{array}$ \\
\hline 34 & $\begin{array}{l}\text { Existen procedimientos para } \\
\text { recoger quejas de proveedores }\end{array}$ & $\begin{array}{c}0.775 \\
(1)\end{array}$ & $\begin{array}{c}0.7262 \\
(1)\end{array}$ & 0.75 & $\begin{array}{c}0.6935 \\
(1)\end{array}$ & $0.6(1)$ \\
\hline 35 & $\begin{array}{l}\text { Estrategias de cooperación con } \\
\text { proveedores }\end{array}$ & $\begin{array}{c}0.6429 \\
(0.5)\end{array}$ & $\begin{array}{c}0.8977 \\
(1)\end{array}$ & 1 & 0.8281 & $0.6(0.5)$ \\
\hline
\end{tabular}

Tabla 8

Pesos por aspecto de las relaciones con proveedores

\begin{tabular}{|c|l|c|c|c|c|c|}
\hline $\begin{array}{c}\text { Rel. } \\
\text { Preg. }\end{array}$ & \multicolumn{1}{|c|}{ Aspecto } & $\begin{array}{c}\text { Constr. y } \\
\text { contratas }\end{array}$ & Fabric. & Transp.* & Mayor. & Minor. \\
\hline 38,31 & Calidad y seguridad de producto & $\begin{array}{c}0.9262 \\
(1)\end{array}$ & $\begin{array}{c}0.9545 \\
(1)\end{array}$ & 0.75 & $\begin{array}{c}0.9194 \\
(1)\end{array}$ & $0.95(1)$ \\
\hline 32,36 & $\begin{array}{l}\text { Efectos medioambientales de los } \\
\text { productos de los proveedores y } \\
\text { su proceso de producción }\end{array}$ & $\begin{array}{c}0.8571 \\
(1)\end{array}$ & $\begin{array}{c}0.7326 \\
(1)\end{array}$ & 0.5 & $\begin{array}{c}0.6897 \\
(0.5)\end{array}$ & $0.85(1)$ \\
\hline 33,37 & $\begin{array}{l}\text { Condiciones laborales de pro- } \\
\text { veedores }\end{array}$ & $\begin{array}{c}0.875 \\
(1)\end{array}$ & $\begin{array}{c}0.6071 \\
(0.5)\end{array}$ & 0.75 & $0.6(0.5)$ & $\begin{array}{c}0.65 \\
(0.5)\end{array}$ \\
\hline 39 & Transparencia financiera & $\begin{array}{c}0.7619 \\
(1)\end{array}$ & $\begin{array}{c}0.75(1) \\
0.5\end{array}$ & $\begin{array}{c}0.6935 \\
(1)\end{array}$ & $0.8(1)$ \\
\hline 34,35 & Respeto por el proveedor & $\begin{array}{c}0.9048 \\
(1)\end{array}$ & $\begin{array}{c}0.8605 \\
(1)\end{array}$ & 0.75 & $0.85(1)$ & $\begin{array}{c}0.75 \\
(0.75)\end{array}$ \\
\hline
\end{tabular}




\subsection{Sociedad}

Hay muchos autores que sostienen que la empresa debe perseguir no sólo el beneficio económico sino también el beneficio social. Es por este motivo que la sociedad como stakeholder en RSC debería jugar un papel importante. Sin embargo, en nuestro estudio la mayoría de los elementos evaluados alcanzaron unos valores no demasiado elevados, con la única excepción de aquellos relacionados directamente con el medioambiente en los que el nivel de desarrollo parece aceptable. No debemos olvidar que muchas empresas empiezan por aspectos medioambientales cuando tratan de aproximarse a una gestión responsable. Los valores alcanzados por los indicadores que miden el desempeño en actividades sociales o en el dialogo con ONG's ponen de manifiesto que queda mucho trabajo por hacer, tanto a nivel de actuación, como de concienciación sobre la importancia que estos elementos tienen.

Tabla 9

Resultados de las relaciones con la sociedad (media y entre paréntesis mediana)

\begin{tabular}{|c|c|c|c|c|c|c|}
\hline $\begin{array}{l}\mathrm{N}^{\circ} \\
\text { Preg. }\end{array}$ & Aspecto & $\begin{array}{l}\text { Constr. y } \\
\text { contratas } \\
\end{array}$ & Fabric. & Transp & Mayor. & Minor. \\
\hline 50 & Informe medioambiental & $0.7381(1)$ & $\begin{array}{l}0.7791 \\
(1)\end{array}$ & 0.5 & $0.4(0)$ & $\begin{array}{c}0.5 \\
(0.5) \\
\end{array}$ \\
\hline 41 & Sin multas medioambientales & nd & $\begin{array}{c}0.8837 \\
(1)\end{array}$ & 1 & $1(1)$ & $1(1)$ \\
\hline 42 & $\begin{array}{l}\text { Los empleados están } \\
\text { preocupados por los estándares } \\
\text { medioambientales }\end{array}$ & $0.85(1)$ & $\begin{array}{l}0.9524 \\
\quad(1)\end{array}$ & 1 & $\begin{array}{l}0.7931 \\
(1)\end{array}$ & $0.9(1)$ \\
\hline 43 & Reducción actual de la polución & $0.8(1)$ & $\begin{array}{l}0.9756 \\
(1)\end{array}$ & 0.5 & $\begin{array}{l}0.7778 \\
(1)\end{array}$ & $1(1)$ \\
\hline 47,44 & $\begin{array}{l}\text { Existe un dialogo activo con } \\
\text { ONG's medioambientales }\end{array}$ & $\begin{array}{l}0.2857,0 \\
\quad(0,0)\end{array}$ & $\begin{array}{c}0.25 \\
(0.25)\end{array}$ & $0.5,0$ & $\begin{array}{l}0.3667 \\
(0.25) \\
0.1333 \\
(0)\end{array}$ & $\begin{array}{l}0.3(0), \\
0(0)\end{array}$ \\
\hline 48,49 & $\begin{array}{l}\% \text { del beneficio gastado en } \\
\text { actividades sociales }\end{array}$ & $\begin{array}{c}0.1905 \\
(0) \\
0.1667(0)\end{array}$ & $\begin{array}{c}0.4861 \\
(0.5)\end{array}$ & $1,-$ & $\begin{array}{l}0.3235 \\
(0), \\
0.1333 \\
(0) \\
\end{array}$ & 0,0 \\
\hline 45 & $\begin{array}{l}\text { Se puede llegar en transporte } \\
\text { público }\end{array}$ & $0.6(1)$ & $\begin{array}{c}0.6098 \\
(1)\end{array}$ & 1 & $0.9(1)$ & $\begin{array}{c}0.889 \\
(1)\end{array}$ \\
\hline 46 & $\begin{array}{l}\text { Existe un plan de uso de } \\
\text { transporte público por parte de } \\
\text { los empleados }\end{array}$ & $0.0714(0)$ & $\begin{array}{l}0.2442 \\
\quad(0)\end{array}$ & 0 & $\begin{array}{l}0.2167 \\
\quad(0)\end{array}$ & $0(0)$ \\
\hline
\end{tabular}


Tabla 10

Pesos por aspecto de las relaciones con la sociedad

\begin{tabular}{|l|l|c|c|c|c|c|}
\hline Rel. Preg. & \multicolumn{1}{|c|}{ Aspecto } & $\begin{array}{c}\text { Constr. y } \\
\text { contratas }\end{array}$ & Fabric. & Transp. & Mayor. & Minor. \\
\hline $\begin{array}{l}50,41, \\
42,43\end{array}$ & Medioambiente & $\begin{array}{c}0.9286 \\
(1)\end{array}$ & $0.881(1)$ & 0.75 & $\begin{array}{c}0.9032 \\
(1)\end{array}$ & $0.9(1)$ \\
\hline 47,44 & $\begin{array}{l}\text { Dialogo activo con ONG's } \\
\text { medioambientales }\end{array}$ & $\begin{array}{c}0.2381 \\
(0)\end{array}$ & $\begin{array}{c}0.2558 \\
(0)\end{array}$ & 0 & $\begin{array}{c}0.3226 \\
(0.5)\end{array}$ & $0.2(0)$ \\
\hline 49,48 & Acciones sociales & $\begin{array}{c}0.5714 \\
(0.5)\end{array}$ & $\begin{array}{c}0.5698 \\
(0.5)\end{array}$ & 0.5 & $\begin{array}{c}0.5938 \\
(0.5)\end{array}$ & $0.6(0.5)$ \\
\hline 45,46 & $\begin{array}{l}\text { Accesibilidad } \\
\text { (no a través de coche) }\end{array}$ & $0.5(0.5)$ & $\begin{array}{c}0.5116 \\
(0.5)\end{array}$ & 0.5 & $\begin{array}{c}0.5625 \\
(0.5)\end{array}$ & $\begin{array}{c}0.55 \\
(0.5)\end{array}$ \\
\hline
\end{tabular}

\subsection{Accionistas}

En el caso de los accionistas en primer lugar, se buscó para las empresas de la muestra el ratio de rentabilidad financiera que recoge la base de datos SABI referido al año 2006. Se eligió la rentabilidad financiera (resultado neto entre capitales propios) porque se asume que es un indicador de la capacidad de la empresa para crear riqueza a favor de sus accionistas (Sánchez Segura, 1994), es decir, es un indicador de rentabilidad que los directivos buscan maximizar en interés de los propietarios.

Tabla 11

Resultados de las relaciones con los accionistas (media y entre paréntesis mediana)

\begin{tabular}{|l|l|c|c|c|c|c|}
\hline \multicolumn{1}{|c|}{$\mathrm{N}^{\text {}}$ Preg. } & \multicolumn{1}{|c|}{ Aspecto } & $\begin{array}{c}\text { Constr. } \mathrm{y} \\
\text { contratas }\end{array}$ & Fabric. & Transp. & Mayor. & Minor. \\
\hline 57 & $\begin{array}{l}\text { Prevención del comercio } \\
\text { interno de acciones }\end{array}$ & $0.9412(1)$ & $\begin{array}{c}0.8049 \\
(1)\end{array}$ & n.d. & $\begin{array}{c}0.7931 \\
(1)\end{array}$ & $1(1)$ \\
\hline 58 & $\begin{array}{l}\text { Comunicación con } \\
\text { accionistas }\end{array}$ & $0.9474(1)$ & $\begin{array}{c}0.738 \\
(1)\end{array}$ & n.d. & $\begin{array}{c}0.931 \\
(1)\end{array}$ & $0.875(1)$ \\
\hline $\begin{array}{l}\text { Info } \\
\text { secundaria }\end{array}$ & $\begin{array}{l}\text { RENTABILIDAD: } \\
\text { Bo. Neto/capital propio* }\end{array}$ & $\begin{array}{c}0.5952 \\
(0.5)\end{array}$ & $\begin{array}{c}0.4205 \\
(0.25)\end{array}$ & $\begin{array}{c}0.5 \\
(0.5)\end{array}$ & $\begin{array}{c}0.5 \\
(0.5)\end{array}$ & $\begin{array}{c}0.65 \\
(0.75)\end{array}$ \\
\hline
\end{tabular}

Los resultados de los distintos indicadores recogidos en la tabla 11 muestran valores bastante elevados en la comunicación con accionistas y en las acciones encaminadas a prevenir el comercio interno de acciones. La rentabilidad de las empresas ostenta valores medios siendo más baja para el sector de fabricación y más elevada para el sector minorista. 
Las empresas consideran en general, muy importante la comunicación con los accionistas y muy residual las acciones destinadas a prevenir el comercio interno de acciones, tal y como reflejan los resultados contenidos en la tabla 12.

Tabla 12

Pesos por aspecto de las relaciones con los accionistas

\begin{tabular}{|l|l|c|c|c|c|c|}
\hline $\begin{array}{l}\text { Rel. } \\
\text { Preg }\end{array}$ & \multicolumn{1}{|c|}{ Aspecto } & $\begin{array}{c}\text { Constr. y } \\
\text { contratas }\end{array}$ & Fabric. & Transp. & Mayor. & Minor. \\
\hline 57 & $\begin{array}{l}\text { Prevención del comercio } \\
\text { interno de acciones }\end{array}$ & $0.125(0)$ & $0.175(0)$ & n.d. & $\begin{array}{c}0.3214 \\
(0)\end{array}$ & $\begin{array}{c}0.0714 \\
(0)\end{array}$ \\
\hline 58 & Comunicación con accionistas & $0.9412(1)$ & $0.6375(0.5)$ & n.d. & $\begin{array}{c}0.8889 \\
(1)\end{array}$ & $\begin{array}{c}0.7143 \\
(1)\end{array}$ \\
\hline
\end{tabular}

\subsection{Competidores}

Las empresas encuestadas consideran capital la libre competencia y las acciones preventivas de sobornos, tal y como reflejan los datos de la tabla 14. Estos aspectos son en los que el trabajo realizado es mayor, ya que se hacen esfuerzos en que no existan quejas sobre la libre competencia o sobre la falta de respeto al capital intelectual de la competencia. En el otro extremo no existen apenas acciones implementadas para prohibir la corrupción o los sobornos en el seno de la empresa, siendo relevantes por bajos, los valores alcanzados en el sector de transportes y en el de construcciones y contratas (ver tabla 13). Por otra parte, también sería importante hacer una labor de concienciación sobre las ventajas de la cooperación entre empresas del mismo sector.

Tabla 13

Resultados de las relaciones con los competidores (media y entre paréntesis mediana)

\begin{tabular}{|l|l|c|c|c|c|c|}
\hline $\begin{array}{c}\mathrm{N}^{\circ} \\
\text { Preg. }\end{array}$ & \multicolumn{1}{|c|}{ Aspecto } & $\begin{array}{c}\text { Constr. y } \\
\text { contratas }\end{array}$ & Fabric. & Transp. & Mayor. & Minor. \\
\hline 52 & $\begin{array}{l}\text { No existen quejas sobre la falta } \\
\text { de respeto al capital intelectual } \\
\text { de la competencia }\end{array}$ & $\begin{array}{c}0.9048 \\
(1)\end{array}$ & $\begin{array}{c}0.9762 \\
(1)\end{array}$ & 0 & $\begin{array}{c}0.9688 \\
(1)\end{array}$ & $1(1)$ \\
\hline 53 & $\begin{array}{l}\text { No existen quejas sobre los } \\
\text { acuerdos que limitan la com- } \\
\text { petencia }\end{array}$ & $1(1)$ & $\begin{array}{c}0.9767 \\
(1)\end{array}$ & 1 & $\begin{array}{c}0.8387 \\
(1)\end{array}$ & $1(1)$ \\
\hline 54 & $\begin{array}{l}\text { La compañía explícitamente } \\
\text { prohíbe la corrupción o los } \\
\text { sobornos }\end{array}$ & $\begin{array}{c}0.1905 \\
(0)\end{array}$ & $\begin{array}{c}0.3256 \\
(0)\end{array}$ & 0 & $\begin{array}{c}0.2097 \\
(0)\end{array}$ & $\begin{array}{c}0.45 \\
(0.25)\end{array}$ \\
\hline 55 & $\begin{array}{l}\text { Cooperación con la compe- } \\
\text { tencia }\end{array}$ & $\begin{array}{c}0.6429 \\
(0.5)\end{array}$ & $\begin{array}{c}0.6707 \\
(0.5)\end{array}$ & 0 & $\begin{array}{c}0.5625 \\
(0.5)\end{array}$ & $\begin{array}{c}0.35 \\
(0.5)\end{array}$ \\
\hline
\end{tabular}


Tabla 14

Pesos por aspecto de las relaciones con los competidores

\begin{tabular}{|l|l|c|c|c|c|c|}
\hline $\begin{array}{c}\text { Rel. } \\
\text { Preg }\end{array}$ & \multicolumn{1}{|c|}{ Aspecto } & $\begin{array}{c}\text { Constr. y } \\
\text { contratas }\end{array}$ & Fabric. & Transp & Mayor. & Minor. \\
\hline 52 & $\begin{array}{l}\text { Respeto al capital intelectual } \\
\text { de la competencia }\end{array}$ & $0.75(1)$ & $0.6707(0.5)$ & 0.5 & $\begin{array}{c}0.6562 \\
(0.5)\end{array}$ & $0.8(1)$ \\
\hline 53 & Libre competencia & $0.85(1)$ & $0.8953(1)$ & 1 & $0.871(1)$ & $0.9(1)$ \\
\hline 55 & $\begin{array}{l}\text { Cooperación con la compe- } \\
\text { tencia }\end{array}$ & $\begin{array}{c}0.7143 \\
(0.5)\end{array}$ & $0.4524(0.5)$ & 0 & $\begin{array}{c}0.5625 \\
(0.5)\end{array}$ & $\begin{array}{c}0.5 \\
(0.5)\end{array}$ \\
\hline 54 & Prevención de sobornos & $0.9286(1)$ & $0.8452(1)$ & 0 & $0.7656(1)$ & $0.95(1)$ \\
\hline
\end{tabular}

Finalmente en la tabla 15 , se recoge un resumen de los resultados medios alcanzados por cada stakeholder. En ella podemos comprobar como las empresas tienen un nivel elevado de desempeño en relación a los clientes, a los que también le otorgan una gran relevancia. Las acciones concernientes a los accionistas alcanzaron valores altos con excepción del sector del transporte. La sociedad es considerada el stakeholder de menor importancia, aunque no para todos los sectores, puesto que para las empresas dedicadas a construcciones y contratas o al sector de fabricación lo menos importante son los accionistas.

Tabla 15

Resumen de resultados por stakeholder, valores medios

\begin{tabular}{|l|l|c|c|c|c|c|c|}
\hline \multicolumn{1}{|c|}{ Stakeholder } & Aspecto & $\begin{array}{c}\text { Constr. y } \\
\text { contratas }\end{array}$ & Fabric. & Transp. & Mayor. & Minor. & Media \\
\hline \multirow{3}{*}{ Clientes } & Desempeño & 0.6765 & 0.7505 & 0.8214 & 0.666 & 0.8214 & 0.7472 \\
\cline { 2 - 8 } & Importancia & 0.9206 & 0.9225 & 0.8333 & 0.8646 & 0.95 & 0.8982 \\
\hline \multirow{3}{*}{ Empleados } & Desempeño & 0.5740 & 0.6619 & 0.6842 & 0.5776 & 0.6169 & 0.6229 \\
\cline { 2 - 8 } & Importancia & 0.8253 & 0.804 & 0.8928 & 0.7879 & 0.8643 & 0.8349 \\
\hline \multirow{3}{*}{ Sociedad } & Desempeño & 0.7787 & 0.7689 & 0.777 & 0.6707 & 0.6611 & 0.7313 \\
\cline { 2 - 8 } & Importancia & 0.865 & 0.7809 & 0.65 & 0.7505 & 0.8 & 0.7693 \\
\cline { 2 - 8 } & Desempeño & 0.5051 & 0.6476 & 0.6875 & 0.5972 & 0.5736 & 0.6022 \\
\hline \multirow{3}{*}{ Accionistas } & Desempeño & 0.8279 & 0.6545 & 0.5 & 0.7414 & 0.8417 & 0.7131 \\
\cline { 2 - 8 } & Importancia & 0.5331 & 0.4062 & nd & 0.6051 & 0.7857 & 0.5825 \\
\hline \multirow{2}{*}{ Competidores } & Desempeño & 0.6845 & 0.7373 & 0.25 & 0.6449 & 0.7 & 0.6033 \\
\cline { 2 - 8 } & Importancia & 0.8107 & 0.7159 & 0.375 & 0.7138 & 0.7875 & 0.6806 \\
\hline
\end{tabular}




\section{CONCLUSIÓN}

Después de la revisión realizada consideramos que es importante tener en cuenta que hasta ahora no existe un estándar aceptado internacionalmente, es decir, dado que los mismos estándares no existen globalmente, los intentos de benchmarking deben reconocer esas diferencias. Así, las empresas necesitan ser comunicativas y explicar como se han desarrollado los benchmarks y porque se seleccionó cada uno de ellos. Por su parte, los stakeholders deben reconocer que el proceso hacia los benchmarks es muy importante, así como la rapidez con la que una empresa alcanza su propio benchmark en relación a los de otras empresas. La comparación no puede realizarse en el vacío, sin sensibilidad hacia las características de la empresa, especialmente hacia los recursos financieros disponibles y la localización. Es decir, el tipo de actividades que deben ser propuestas por la empresa dependen en gran medida de su condición financiera, tamaño, localización, productos o servicios, restricciones legales y la dedicación de la dirección y el equipo directivo. El estándar mínimo interno de benchmarking debe reflejar claramente los requerimientos legales. Desafortunadamente, muchas empresas (especialmente las multinacionales) operan bajo múltiples jurisdicciones legales que tienen diferentes puntos de vista sobre el comportamiento apropiado, y la legalidad puede variar de una localización a otra. En esas circunstancias, el benchmarking mínimo interno debe ser el estándar legal más elevado de todas las localizaciones y ese estándar debe cumplirse con independencia de la localización.

En este trabajo y siguiendo a Graafland et al (2004) se ha propuesto un sistema de indicadores que evalúa hasta 68 aspectos de RSC, agrupados en seis stakeholders. También se ha preguntado a las empresas de cinco sectores por la importancia que otorgan a estos aspectos. Con la información recopilada en indicadores de desempeño homogéneos por aspecto y de importancia (rango 0 a 1) es posible establecer comparaciones entre distintas empresas o a nivel sectorial. También se pueden detectar aquellas áreas en las que el trabajo realizado es menor o que necesitan de una mayor concienciación puesto que han obtenido puntuaciones de importancia más bajas.

Tal y como se ha puesto de manifiesto en epígrafes anteriores, los resultados alcanzados han revelado que el stakeholder al que de media se le está otorgando mayor importancia, evaluada a través de acciones que han sido puestas en funcionamiento por las empresas es el accionista. También hay un elevado nivel de desempeño general con los clientes, siendo estos a los que se les considera un stakeholder fundamental para la supervivencia de la empresa. Para el resto de stakeholders no se ha detectado un patrón único, y tampoco se han encontrado diferencias sustanciales en función del sector. En la mayoría de los casos, suele existir un elevado nivel de actuación en los aspectos que se hayan recogidos en algún tipo de normativa obligatoria o voluntaria, y un menor nivel en aquellos otros asociados a una estrategia clara de apuesta por la RSC. Por ello, podemos derivar que la RSC en las empresas analizadas a nivel general está en un nivel iniciático, se cumplen algo más que los requisitos mínimos pero se hace necesario avanzar en algunos aspectos directamente relacionados con este nuevo enfoque estratégico. Sin embargo, algo bueno subyace puesto que existen algunas empresas que están siendo pioneras dentro de su sector liderando este camino de apuesta por la RSC. 


\section{BIBLIOGRAFÍA}

ARAQUE PADILLA, R.; MONTERO SIMÓ, M.J. (2005): "Una propuesta de sistematización del marco de relaciones de las entidades no lucrativas: un enfoque basado en los stakeholders", Icade: Revista de las Facultades de Derecho y Ciencias Económicas y Empresariales, ${ }^{\circ} 65$, pp. 111-134.

ARAQUE PADILLA, R. (2009): "Empresa y sociedad: el enfoque de la responsabilidad social de la empresa", Revista de Fomento Social, no 256, pp. 679-686.

ARGANDOÑA, A y VON WEILTZIEN HOIVIK, H (2009): "Corporate Social Responsibility: One size does not fit all. Collecting evidence from Europe", Journal of Business Ethics, №. 89, pp. 221-234

BAJO DAVO, N.; DURÁN HERRERA, J.J. (2009): "Responsabilidad social y variables estratégicas en las grandes empresas españolas", Revista de responsabilidad social en la empresa, $\mathrm{n}^{\circ} 2$, pp. 51-76.

CAMPBELL, L.; GULAS, C. S. y GRUCA, T. S. (1999): "Corporate Giving Behavior and DecisionMaker Social Consciousness", Journal of Business Ethics, No. 19, pp.375-383.

COMISIÓN DE LAS COMUNIDADES EUROPEAS (2001): Libro verde. Fomentar un marco europeo para la responsabilidad social de las empresas. Disponible en, http://europa.eu/eur-lex/es/ com/gpr/2001/com2001_0366es01.pdf

DONALDSON, T. y PRESTON, L. R. (1995): "The Stakeholder Theory of the Corporation: Concepts, Evidence and Implications", Academy of Management Review, Vol. 20, pp. 65-91.

FORÉTICA (2006): Evolución de la responsabilidad social de las empresas en España. (Disponible en www.foretica.es).

GLOBAL REPORTING INITIATIVE (2002): Final report of the measurement working group. Disponible en, http://www.globalreporting.org

GRAAFLAND, J.J. y EIJFFINGER, SCW (2004): "Corporate social responsibility of Dutch companies: Benchmarking, transparency and robustness", De Economist, $\mathrm{n}^{\circ}$ 152, pp. 403-426.

GRAAFLAND, J.J. y EIJFFINGER, SCW y SMID, H. (2004): "Benchmarking of Corporate Social Responsibility: Methodological Problems and Robustness", Journal of Business Ethics, $\mathrm{n}^{\circ}$ 53, pp. 137-152.

GRAAFLAND, JJ (2002): "Modelling the trade off between profits and principles", De Economist, $n^{\circ} 150$, pp. 129-154.

GRAVES, SB; WADDOCK, SA y KELLY, J (2002): “100 best corporate citizens”, Business Ethics, vol. $11, n^{\circ} 2$, pp. 8-13.

HILLMAN, A. J. y KEIM, G. D. (2001): "Shareholder Value, Stakeholder Management, and Social Issues: What's the Bottom Line?", Strategic Management Journal, Vol. 22, pp. 125-139.

IGALENS, J y GOND, J-P (2005): "Measuring Corporate Social Performance in France: A critical and empirical analysis of ARESE data", Journal of Business Ethics, $\mathrm{N}^{\circ}$ 56, pp. 131-148

INTERNATIONAL CHAMBER OF COMMERCE (2002): Business in Society: Making a positive and responsible contribution. ICC: Paris.

JOYNER, B. E. y PAYNE, D. (2002): "Evolution and Implementation: A Study of Values, Business Ethics and Corporate Social Responsibility”, Journal of Business Ethics, Vol. 41, pp. 297-311.

JOYNER, B.E. y RAIBORN, CA (2005): "Management caveats for measuring and assessing public responsibility performance", Business horizons, $\mathrm{n}^{\circ}$. 48, 525-533

KOLODINSKY, RW; MADDEN, TM; ZISK, DS y HENKEL, ET (2010): “Attitudes about Corporate Social Responsibility: Business student predictors", Journal of Business Ethics, $\mathrm{N}^{\circ}$. 91, pp. 167-181.

KRUT, R y MUNIS, K (1998): "Sustainable industrial development: benchmarking environmental policies and reports", Greener Management International, $\mathrm{n}^{\circ}$ 21, pp. 88-98. 
LANTOS, G. P. (2001): “The Boundaries of Strategic Corporate Social Responsibility”, Journal of Consumer Marketing, Vol. 18, pp. 595-630.

MARGOLIS, J. D. y WALSH, J. P. (2003): "Misery Loves Companies: Rethinking Social Initiatives by Business", Administrative Science Quarterly, Vol. 48, pp. 268-305.

MCWILLIAMS, A. y SIEGEL, D. (2001): "Corporate Social Responsibility: A Theory of the Firm Perspective", Academy of Management Review, Vol. 26, pp. 117-127.

ORLITZKY, M.; SCHMIDT, F. L. y RYNES, S. L. (2003): “Corporate Social and Financial Performance: A Meta-Analysis", Organization Studies, Vol. 24, pp. 403-441.

PORTER, ME y KRAMER, MR (2002): "The competitive advantage of corporate philanthropy", Harvard Business Review, vol. 80, $\mathrm{n}^{\circ}$ 12, pp. 56-69.

RUSCONI, G. (2002): "The theory and practise of social accounting: the case of the Italian post office", paper presentado en la EBEN Conference en Bruselas.

TENCATI, A.; PERRINI, F. y POGUTZ, S. (2004): "New tools to foster corporate socially responsible behavior", Journal of Business Ethics, $\mathrm{n}^{\circ}$ 53, pp. 173-190.

VALOR, C. (2005): "Corporate Social Responsibility and Corporate Citizenship: Towards Corporate Accountability", Business and Society Review, vol. 110, n 2, pp. 191-212.

WADDOCK, S. A. y GRAVES, S. B. (1997): "The Corporate Social Performance-Financial Performance Link", Strategic Management Journal, vol. 18, pp. 303-319. 


\section{Gipuzkoaren kanpo harremanak, esku onetan.}

Las relaciones externas de Gipuzkoa, en buenas manos.

Les relations externes de Gipuzkoa, en bonnes mains.

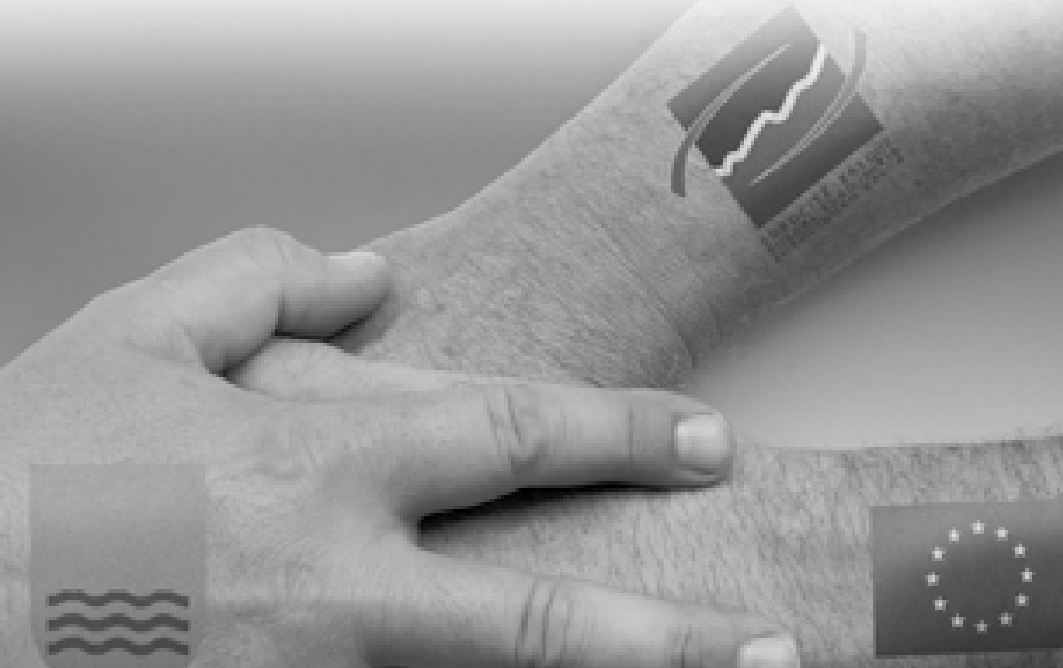

